\title{
Incidence des traitements technologiques sur la qualité hygiénique du lait et des fromages de chèvre à pâte molle
}

\author{
MF Gay, G Jaubert, S Saboureau
}

Institut technique des produits laitiers caprins, BP 49, 17700 Surgères, France

\begin{abstract}
Résumé - Cette étude avait un triple objectif : comparer l'efficacité des traitements d'épuration bactérienne du lait de chèvre, comparer le comportement du lait cru et des laits épurés pendant l'acidification, et étudier le développement de la flore staphylocoque et des coliformes fécaux pendant l'élaboration de fromages à pâte molle suivant 2 technologies (prise lente et prise rapide). Les traitements de pasteurisation $\left(70^{\circ} \mathrm{C}, 5 \mathrm{~min}\right)$ et de microfiltration $(1,4 \mu \mathrm{m})$ ont un effet très significatif sur le niveau de contamination du lait : ils permettent respectivement de réduire la flore staphylocoque de 1,4 et 1,1 unités logarithmiques $(P<0,001)$, et les coliformes fécaux de $1,4(P<0,01)$ et 1,0 $(P<0,05)$ unités. Pendant la maturation à $22^{\circ} \mathrm{C}$, le développement de ces flores est inversement proportionnel à celui de la flore lactique. L'acidification du lait microfiltré est moins importante que celle du lait pasteurisé, elle-même plus faible que celle du lait cru. Les niveaux de contamination observés après coagulation et égouttage sont supérieurs à ceux du lait de chèvre mis en œuvre ; les populations en coliformes fécaux et staphylocoques présentes dans les fromages âgés de $30 \mathrm{j}$ sont comparables à celles observées au démoulage. La maîtrise d'un faible niveau de contamination en coliformes fécaux du lait de chèvre permet de garantir un niveau de population acceptable dans les fromages à pâte molle, quelle que soit la technologie utilisée ; la recherche d'un développement optimal de la flore lactique acidifiante pendant la maturation apparaît indispensable pour la maîtrise du niveau de contamination en staphylocoques des fromages, notamment en technologie prise rapide.
\end{abstract}

lait de chèvre / fromage de chèvre / pasteurisation / microfiltration / staphylocoques / coliformes fécaux

Summary - Effect of pasteurization and microfiltration on the hygienic quality of goat's milk and on soft goat's cheeses. The aims of this study were firstly to compare the reduction in contaminant flora when pasteurization $\left(70^{\circ} \mathrm{C}\right.$ for $\left.5 \mathrm{~min}\right)$ or microfiltration $(1.4 \mu \mathrm{m}$ pore size) are applied to goat's milk, secondly the acidification kinetics of both milks compared to that of raw milk, and thirdly the development of contaminant faecal coliforms and staphylococci during the making and ripening of 2 types of goat's cheese: lactic and rennet curds. Pasteurization and microfiltration were found to significantly reduce the contaminant flora in goat's milk. Staphylococcal flora were reduced respectively by 1.4 and $1.1 \mathrm{Log}$ units $(\mathrm{P}<0.001)$ and faecal coliforms by 1.4 and $1.0 \mathrm{Log}$ units $(\mathrm{P}<0.05)$ respectively. Development of contaminant flora during milk acidification at $22^{\circ} \mathrm{C}$ was inversely proportional to the initial level of lactic flora. Acidification rate was highest in raw milk, intermediate in pasteurized milk and lowest in microfiltrated milk. Both pasteurized and microfiltrated milks allowed a higher development of contaminant flora than raw milk in the drained curds; these differences were 
also observed in 30-d-old ripened cheeses manufactured from the 3 types of milks. Pasteurization and microfiltration are processes which guarantee a low level of staphylococci and faecal coliforms in soft goat's cheese but research into the optimal growth of lactic acid starter during ripening must be carried out, particularly for rennet curd cheese technology.

goat's milk / goat's cheese / pasteurization / microfiltration / Staphylococcus / faecal coliforms

\section{INTRODUCTION}

La population microbienne présente dans un lait de chèvre destiné à la transformation fromagère peut être très variable, aussi bien en quantité qu'en qualité. La nature et le niveau des contaminations sont liés à de nombreux facteurs - état sanitaire du troupeau (Le Guillou, 1989), hygiène et conditions de traite, conditions de stockage à la ferme et à l'usine, conditions de collecte - et peuvent se traduire par une altération des propriétés technologiques du lait ou de la qualité hygiénique des produits obtenus.

L'assainissement thermique du lait de chèvre collecté est une pratique industrielle courante. Toutefois, compte tenu de sa sensibilité particulière à la chaleur (Zadow et al, 1983), les températures de traitement pratiquées restent modérées (pasteurisation basse, thermisation). Par ailleurs, la microfiltration du lait préalablement écrémé sur membranes $1,4 \mu \mathrm{m}$ est un procédé d'épuration efficace, d'autant plus intéressant pour le lait de chèvre que le traitement est réalisé à une température inférieure à $50^{\circ} \mathrm{C}$ (Jaubert et al, 1991).

Le développement des flores de contamination pendant la transformation fromagère, et par conséquent la qualitè hygiénique des fromages, est non seulement liée au niveau de contamination du lait mis en œuvre, mais également aux paramètres technologiques utilisés. L'effet déterminant de l'acidification et de la température sur le développement dés bactéries indésirables lors de la fabrication de fromages à pâte molle au lait de vache a été démontré (Mourgues et al, 1977 ; Richard et Gratadoux, 1986).

Dans ce contexte, le présent travail avait pour objectif d'une part de comparer l'efficacité des traitements d'épuration (thermisation, microfiltration) appliqués au lait de chèvre, d'autre part de comparer le comportement des laits ainsi épurés pendant l'acidification fromagère, et enfin d'étudier l'évolution des flores contaminantes (staphylocoques présumés pathogènes, coliformes fécaux) pendant la fabrication de fromages de chèvre à pâte molle.

\section{MATÉRIEL ET MÉTHODES}

\section{Lait utilisé}

Le lait de chèvre utilisé était un lait entier, cru, de petit mélange collecté dans la région de Surgères toutes les $48 \mathrm{~h}$ et acheminé au laboratoire.

\section{Épuration bactérienne des laits}

Pour chaque essai, 90 I de lait de chèvre étaient répartis en 3 lots : le premier subissait une pasteurisation en cuve, $70^{\circ} \mathrm{C}, 5 \mathrm{~min}$, puis était refroidi. Le deuxième lot était réchauffé à $40^{\circ} \mathrm{C}$ et écrémé par centrifugation : le lait écrémé était épuré en continu à $40^{\circ} \mathrm{C}$, à l'aide d'un matériel pilote de microfiltration Alfa-Laval type MFS1 (Les Clayes-sous-Bois, France), suivant les conditions décrites par Jaubert et al (1991) ; la crème, dont le volume représentait $10 \%$ du volume de lait entier, subissait une pasteurisation en cuve, $90^{\circ} \mathrm{C}, 3 \mathrm{~min}$, avant d'être refroidie et ré- 
incorporée au lait écrémé épuré. Le troisième lot ne subissait aucun traitement d'épuration.

Huit essais ont permis d'étudier l'efficacité des traitements d'épuration sur la flore coliforme fécale et la flore staphylocoque présumée pathogène. Les échantillons de lait des 3 lots étaient prélevés stérilement et immédiatement refroidis à une température inférieure à $4^{\circ} \mathrm{C}$. Les dénombrements bactériens étaient effectués dans les 2 h suivant le prélèvement.

\section{Étude du comportement des laits épurés pendant l'acidification}

Quatre essais ont permis de comparer les comportements des laits cru, pasteurisé ou microfiltré pendant l'acidification. L'acidification était réalisée en flacons stériles de $1 \mathrm{I}$. Deux flacons ont été prélevés pour chacun des 3 lots de laits : le premier était ensemencé en flore lactique mésophile (CHN12, Boll) à raison de $2 \%$, puis incubé $24 \mathrm{~h}$ dans un bain-marie à $22^{\circ} \mathrm{C}$; le second était placé directement à $22^{\circ} \mathrm{C}$.

Le pH et l'acidité ont été mesurés à différents stades de maturation : au départ $(\mathrm{HO})$, après $1 \mathrm{~h}$ (H1), $3 \mathrm{~h}(\mathrm{H} 3), 6 \mathrm{~h}(\mathrm{H} 6), 12 \mathrm{~h}(\mathrm{H} 12), 18 \mathrm{~h}(\mathrm{H} 18)$, $21 \mathrm{~h}(\mathrm{H} 21)$ et $24 \mathrm{~h}(\mathrm{H} 24)$ pour l'ensemble des échantillons, et après $30 \mathrm{~h}(\mathrm{H} 30), 36 \mathrm{~h}(\mathrm{H} 36)$, $42 \mathrm{~h}(\mathrm{H} 42)$ et $48 \mathrm{~h}(\mathrm{H} 48)$ pour les laits non ensemencés.

La phase de latence de production d'acide lactique a été définie comme étant la durée (en heures) nécessaire pour produire $0,2 \mathrm{~g}$ d'acide lactique/l de lait.

Les dénombrements des flores totale, lactique, psychrotrophe, coliforme et staphylocoque présumée pathogène ont été réalisés à $\mathrm{HO}$ et H24, pour les échantillons ensemencés, à $\mathrm{HO}$ et H48 pour les échantillons non ensemencés. Les taux multiplicatifs ont été calculés pour les différentes flores, après $24 \mathrm{~h}$ pour les échantillons ensemencés et après $48 \mathrm{~h}$ pour les échantillons non ensemencés.

Le traitement des données a été réalisé à l'aide du logiciel STATITCF (ITCF, Boigneville, France). Une analyse en composantes principales (ACP) a permis d'une part d'étudier les relations entre les différentes variables mesurées, et, d'autre part, de comparer le comportement des différents échantillons pendant la maturation.

\section{Évolution des flores contaminantes pendant la transformation fromagère}

Huit essais ont permis de mesurer l'évolution des flores coliforme fécale et staphylocoque présumée pathogène pendant la transformation fromagère, en fonction du niveau de contamination initial et de la technologie utilisée.

Pour chacun des essais, 15 I des 3 laits (cru, microfiltré ou pasteurisé) étaient transformés en fromages à pâte molle type bûchettes (prise lente) et 15 autres litres en fromages à pâte molle type chèvre boîte (prise rapide). Pour les fabrications de type prise lente, la température du lait était réglée à $22^{\circ} \mathrm{C}$. Un ensemencement en ferments lactiques mésophiles (CHN12) était réalisé à raison de $2 \%$. Après une maturation permettant d'atteindre un $\mathrm{pH}$ de 6,4 , le lait était emprésuré à une dose de $5 \mathrm{ml} / 100$ I d'enzyme contenant $520 \mathrm{mg} / \mathrm{l}$ de chymosine (Présure Boll). Après coagulation et acidification pendant $18 \mathrm{~h}$, le caillé $(\mathrm{pH} 4,4)$ était moulé à la louche : l'égouttage durait $20 \mathrm{~h}$ à $18^{\circ} \mathrm{C}$; les fromages étaient ensuite démoulés, salés à sec et placés en salle de ressuyage durant $24 \mathrm{~h}\left(11^{\circ} \mathrm{C}, 90 \%\right.$ HR). Après $10 \mathrm{j}$ d'affinage, ils étaient refroidis à $7^{\circ} \mathrm{C}$, emballés et stockés en chambre froide.

Pour les fabrications de type prise rapide, la température du lait était réglée à $34^{\circ} \mathrm{C}$, avant ensemencement en ferments lactiques mésophiles $(2 \%)$. L'emprésurage était réalisé à $\mathrm{pH}$ 6,2 à raison de $18 \mathrm{ml}$ de présure pour $100 \mathrm{l}$; le temps de coagulation était de $2 \mathrm{~h}$, avant découpage et moulage du caillé ; 3 retournements étaient réalisés pendant l'égouttage ; les fromages étaient démoulés à pH 4,8. Les opérations de salage, de ressuyage et d'affinage étaient identiques à celles décrites pour les fromages type prise lente.

Les échantillons étaient prélevés à différents stades de la transformation : lait en cuve avant ensemencement, fromages au démoulage, après ressuyage, après 5 à 6 j d'affinage, à l'emballage, après $3 \mathrm{j}$ et $25 \mathrm{j}$ de stockage en chambre froide à $6^{\circ} \mathrm{C}$.

\section{Analyses microbiologiques}

Le dénombrement de la flore mésophile aérobie révivifiable a été réalisé après $72 \mathrm{~h}$ d'incubation 
à $30^{\circ} \mathrm{C}$, sur milieu Plate Count Agar (FIL $100 \mathrm{~A}$, 1987).

Les coliformes totaux et fécaux ont été dénombrés sur gélose biliée au rouge neutre et au cristal violet (VRBLA) après $24 \mathrm{~h}$ d'incubation respectivement à une température de $30^{\circ} \mathrm{C}$ et $44^{\circ} \mathrm{C}$ (FIL 73A, 1985).

La flore staphylocoque présumée pathogène était mesurée par dénombrement des colonies de couleur noire brillante caractéristique, présentant un halo opaque, sur milieu Baird Parker additionné de tellurite de potassium et jaune d'œuf, après $48 \mathrm{~h}$ d'incubation à $37^{\circ} \mathrm{C}$.

La flore psychrotrophe était dénombrée après $10 \mathrm{j}$ d'incubation à $70^{\circ} \mathrm{C}$ sur milieu Plate Count Agar.

La flore lactique totale était dénombrée sur milieu Elliker après $3 \mathrm{j}$ d'incubation à $30^{\circ} \mathrm{C}$.

Les taux multiplicatifs (TM) ont été calculés à partir des résultats des dénombrements effectués pour les différentes flores:

$$
T M=\log \frac{N}{N_{0}}
$$

avec $\mathrm{N}=$ population finale $(\mathrm{UFC} / \mathrm{ml}) ; \mathrm{N}_{0}=$ population initiale (UFC/ml).

\section{Analyses physico-chimiques}

L'acidité des laits a été déterminée par neutralisation de $10 \mathrm{ml}$ de lait à l'aide d'hydroxyde de sodium $(0,111 \mathrm{~N})$ en présence de phénolphtaléine.
Les mesures de $\mathrm{pH}$ ont été réalisées à l'aide d'un appareil type WTW pH meter 196 (Bioblock, France). Les échantillons de fromages étaient préalablement broyés : $10 \mathrm{~g}$ étaient mis en suspension dans $50 \mathrm{ml}$ d'eau déminéralisée à $50^{\circ} \mathrm{C}$ à l'aide d'un mélangeur ULTRATURRAX. La mesure du pH était effectuée lorsque la solution avait atteint une température de $20^{\circ} \mathrm{C}$.

La quantité d'acide lactique produite était calculée par différence entre les valeurs d'acidité finale et initiale.

\section{RÉSULTATS ET DISCUSSION}

\section{Efficacité des traitements d'épuration vis-à-vis des staphylocoques et des coliformes fécaux}

Les populations résiduelles observées dans les laits épurés (tableau I) sont en moyenne inférieures à $10 \mathrm{UFC} / \mathrm{ml}$ et ne sont pas significativement différentes pour les flores staphylocoque et coliforme fécale. Ces résultats sont comparables à ceux obtenus par Jaubert et al (1991) lors de l'épuration par microfiltration de lait de chèvre écrémé, et sont en accord avec les observations de Portmann et Pierre (1973) qui ont montré qu'une pasteurisation, $72^{\circ} \mathrm{C}, 15 \mathrm{~min}$, permettait de réduire dans $80 \%$ des cas la population coliforme du lait de chèvre à moins de 1 germe/ml.

Tableau I. Niveau moyen de contamination en staphylocoques et en coliformes fécaux (UFC/ml) des laits de chèvre crus, pasteurisés $\left(70^{\circ} \mathrm{C}, 5 \mathrm{~min}\right)$ et épurés par microfiltration $(1,4 \mu \mathrm{m})$. Moyennes $(\mathrm{m})$ et écarts types $(\sigma)$ calculés à partir des résultats de 16 observations.

Mean contamination level of staphylococci and faecal coliforms $(\mathrm{CFU} / \mathrm{ml})$ in raw, pasteurized $\left(70^{\circ} \mathrm{C}\right.$ for $5 \mathrm{~min})$ and microfiltrated $(1.4 \mu \mathrm{m})$ goat's milk. Mean $(\mathrm{m})$ and standard deviations $(\sigma)$ have been calculated from 16 observations.

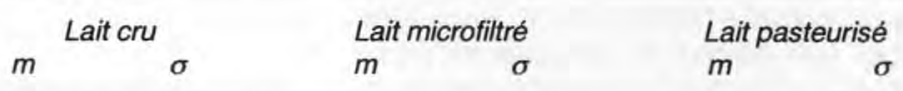

$\begin{array}{lllllll}\text { Coliformes fécaux } & 1,45 & 1,30 & 0,53 & 0,84 & 0,08 & 0,35 \\ \text { Staphylocoques } & 1,97 & 1,00 & 0,92 & 1,04 & 0,60 & 0,80\end{array}$


Les réductions bactériennes moyennes observées sont respectivement voisines de 1,0 et 1,4 unités logarithmiques lors de la microfiltration et la pasteurisation, quelle que soit la flore considérée, ce qui correspond à l'élimination de $91 \%$ et $96 \%$ des coliformes fécaux et des staphylocoques présents dans le lait cru ; l'efficacité des 2 traitements d'épuration étudiés est comparable.

\section{Comportement du lait de chèvre pendant la maturation à $22^{\circ} \mathrm{C}$}

L'analyse des données relatives à l'étude du comportement des laits de chèvre crus et épurés pendant la maturation, (fig 1) permet de distinguer 3 groupes de variables (fig 1.1):

- les niveaux de population initiale des flores totale et lactique des laits étudiés sont étroitement liés $(r=0,97)$ et varient dans le même sens ;

- les taux multiplicatifs des flores lactique et coliforme, la population coliforme finale. et la durée de la phase de latence de production d'acide lactique sont liés $(r>0,90)$, et sont d'autant plus importants que la flore lactique du lait mis en cuvre est faible ;

- les contaminations initiales en coliformes, staphylocoques et psychrotrophes sont liées $(r>0,85)$ et indépendantes des 2 autres groupes de variables.

Par ailleurs, la quantité d'acide lactique maximale produite pendant la maturation du lait de chèvre à $22^{\circ} \mathrm{C}$ est d'autant plus importante que la population initiale en bactéries lactiques est élevée $(r=0,72)$ et que la durée de la phase de latence, les taux multiplicatifs des flores lactiques, coliforme et psychrotrophe sont faibles ( $r<$ $-0,68$ ). Le développement des coliformes est lié au niveau de population initial de la flore lactique $(r=-0,96)$ et à la durée de la phase de latence pendant l'acidification $(r=0,93)$. Le développement de la flore staphylocoque est lié aux mêmes facteurs, mais de façon moins étroite $(r<0,75)$.

L'étude des différents échantillons a permis d'une part de caractériser des laits crus, pasteurisés et microfiltrés, et, d'autre part, de comparer leur comportement pendant l'acidifcation (fig 1.2).

Les laits microfiltrés et pasteurisés présentent des niveaux de contamination en coliformes fécaux et en staphylocoques comparables et plus faibles que ceux observés sur le lait cru ; cela confirme l'efficacité des traitements d'épuration réalisés.

Le comportement des laits épurés non ensemencés est caractérisé par une phase de latence importante lors de l'acidification, ainsi que par des taux multiplicatifs élevés pour les flores lactique et coliforme : le niveau de contamination en germes coliformes est proche de $10^{8}$ UFC/ $\mathrm{ml}$ après $48 \mathrm{~h}$ d'incubation à $22^{\circ} \mathrm{C}$.

L'ensemencement du lait en bactéries lactiques mésophiles se traduit par un démarrage rapide de l'activité acidifiante et un développement limité de la flore coliforme pendant la maturation. Cela est à mettre en relation avec les phénomènes d'antagonisme et d'inhibition, spécifiques ou non, décrits par Catteau (1991), qui interviendraient entre la flore lactique et la flore contaminante.

L'ensemencement du lait en ferments lactiques et le type de traitement d'épuration subi ont une incidence hautement significative $(P<0,001)$ sur la quantité d'acide lactique produite après $24 \mathrm{~h}$ d'incubation à $22^{\circ} \mathrm{C}$ (tableau II).

L'addition de $2 \%$ de bactéries lactiques mésophiles au lait de chèvre se traduit, après incubation à $22^{\circ} \mathrm{C}$, par une multiplication par 20 de la vitesse d'acidification. L'acidification du lait microfiltré est significativement moins importante en moyenne que celle observée sur le lait pasteurisé, qui est elle-même plus faible que celle 


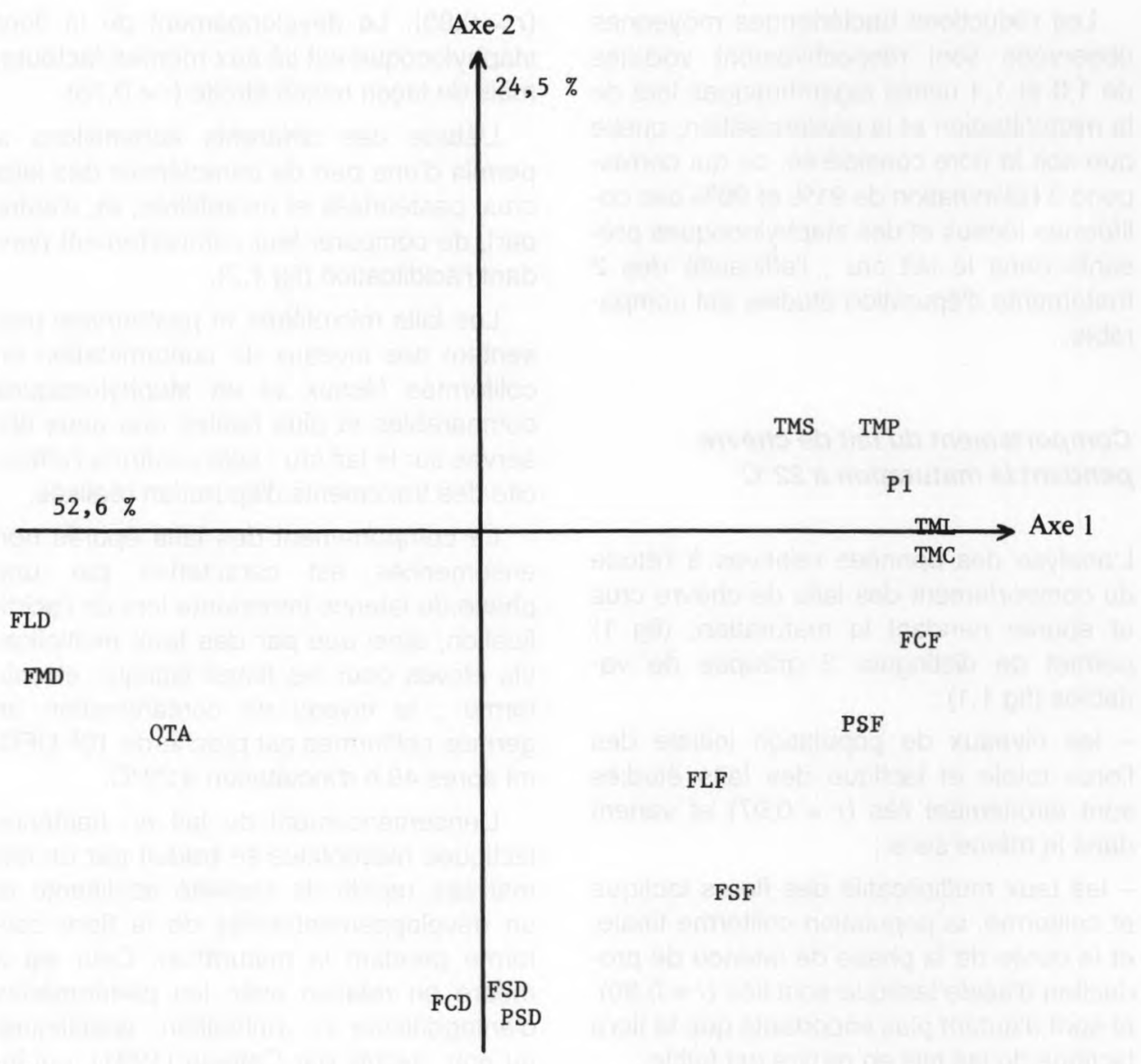

\section{1}

Fig 1. Étude du comportement du lait de chèvre pendant la maturation à $22^{\circ} \mathrm{C}$ (analyse en composantes principales). 1.1. Étude des variables. 1.2. Étude des individus.

Variables étudiées : FMD : flore mésophile totale, population initiale ; PSD : flore psychrotrophe, population initiale ; PSF : flore psychrotrophe, population finale ; FLD : flore lactique, population initiale ; FLF : flore lactique, population finale ; FCD : flore coliforme, population initiale ; FCF : flore coliforme, population finale ; FSD: flore staphylocoque, population initiale; FSF : flore staphylocoque, population finale ; TMP, TML, TMC, TMS : taux multiplicatifs des flores psychrotrophe, lactique, coliforme et staphylocoque ; QTA : quantité d'acide lactique maximale produite $(\mathrm{g} / \mathrm{l})$; $\mathrm{P} 1$ : durée de la phase de latence pendant l'acidification (en h). Les différentes flores sont exprimées en logarithme (UFC/ml).

Observations réalisées : 5 à 8 : laits crus non ensemencés (LC) ; 13 à 16 : laits crus ensemencés (LCE) ; 9 à 12 : laits pasteurisés non ensemencés (LP) ; 21 à 24 : laits pasteurisés ensemencés (LPE) ; 1 à 4 : laits microfiltrés non ensemencés (LM); 17 à 20 : laits microfiltrés ensemencés (LME). 


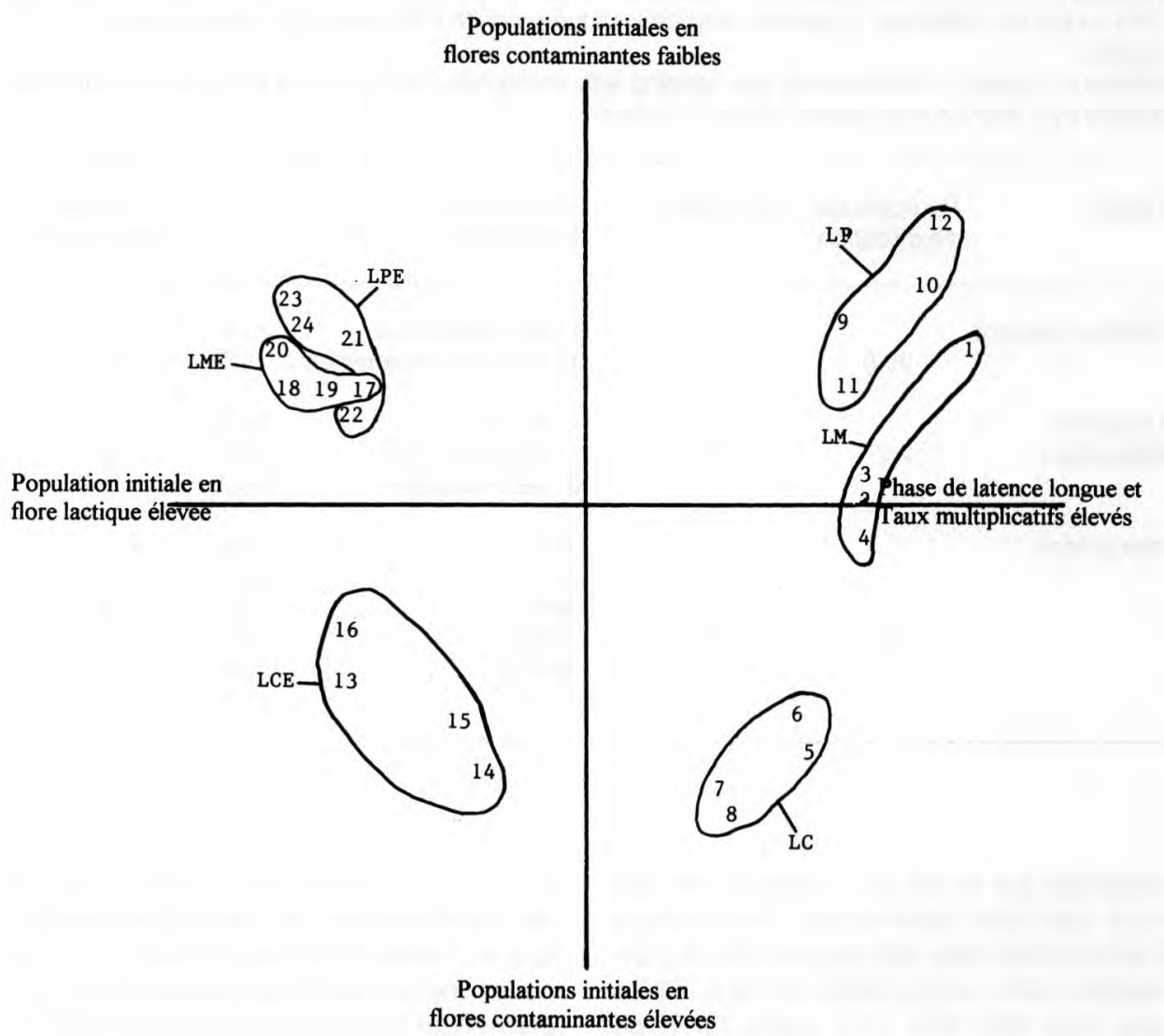

Goat's milk flora during maturation at $22^{\circ} \mathrm{C}(A C P)$. 1.1. Projection of variables on the first and second principal components. 1.2. Projection of samples on the first and second principal components.

Studied variables: FMD: total mesophilic flora, initial population (Log CFU/mI) ; PSD: psychrotrophic flora, initial population (Log CFU/ml) ; PSF: psychrotrophic flora, final population (Log CFU/ml) ; FLD: lactic flora, initial population (Log CFU/ml) ; FLF: lactic flora, final population (Log CFU/ml) ; FCD: coliform flora, initial population (Log CFU/ml) ; FCF: coliform flora, final population (Log CFU/ml) ; FSD: Staphylococcus flora, initial population (Log CFU/mI) ; FSF: Staphylococcus flora, final population (Log CFU/mi) ; TMP, TML, TMC, TMS: multiplicative coefficients of psychrotrophic, lactic, coliform and Staphylococcus flora. QTA: maximum amount of lactic acid produced. P1: lag phase during acidification (h). Observations: 5-8: raw milk (LC) ; 13-16: acidified raw milk (LCE) ; 9-12: pasteurized milk (LP) ; 2124: acidified pasteurized milk (LPE) ; 1-4: microfiltrated milk (LM) ; 17-20: acidified microfiltrated milk (LME). 
Tableau II. Incidence du traitement d'épuration et de l'ensemencement en flore lactique mésophile $(2 \%)$ du lait de chèvre sur la quantité d'acide lactique produite $\left({ }^{\circ} \mathrm{D}\right)$ après $24 \mathrm{~h}$ d'incubation à $22^{\circ} \mathrm{C}(4$ essais).

Influence of goat's milk epuration and seeding with mesophilic lactic bacteria (2\%) on lactic acid production $\left({ }^{\circ} \mathrm{D}\right)$ after $24 \mathrm{~h}$ incubation at $22^{\circ} \mathrm{C}(4 \mathrm{trials})$.

\begin{tabular}{|c|c|c|c|c|c|}
\hline Facteur & $\begin{array}{l}\text { Pourcentage } \\
\text { d'explication }\end{array}$ & Signification & $\begin{array}{l}\text { Comparaison } \\
\text { de moyenne }\end{array}$ & & $\begin{array}{c}\text { Groupes } \\
\text { homogènes }\end{array}$ \\
\hline Ensemencement & 95,8 & $\star \star \star *$ & $\begin{array}{l}\mathrm{E}: \text { lait ensemencé } \\
\mathrm{NE} \text { : lait non ensemencé }\end{array}$ & $\begin{array}{r}64,6 \\
6,0\end{array}$ & $B^{A}$ \\
\hline $\begin{array}{l}\text { Traitement } \\
\text { d'épuration }\end{array}$ & 2,2 & $* * *$ & $\begin{array}{l}\mathrm{C} \text { : lait cru } \\
\mathrm{P} \text { : lait pasteurisé } \\
\mathrm{M} \text { : lait microfiltré }\end{array}$ & $\begin{array}{l}40,6 \\
35,6 \\
29,7\end{array}$ & ${ }^{A} \mathrm{C}$ \\
\hline Interactions & 1,4 & $\star * \star *$ & $\begin{array}{l}\text { LP-E } \\
\text { LC-E } \\
\text { LM-E } \\
\text { LC-NE } \\
\text { LM-NE } \\
\text { LP-NE }\end{array}$ & $\begin{array}{r}70,0 \\
67,1 \\
56,7 \\
14,1 \\
2,6 \\
1,3\end{array}$ & 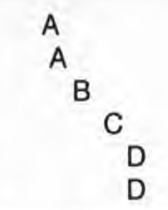 \\
\hline
\end{tabular}

observée sur le lait cru : lorsque les laits n'ont pas été ensemencés, les niveaux d'acidification des laits microfiltrés et pasteurisés sont comparables et plus faibles que ceux des laits crus après $24 \mathrm{~h}$ de maturation ; par contre, après ensemencement, le niveau d'acidification des laits microfiltrés est significativement moins important que celui des laits crus et pasteurisés.

Ces comportements différents correspondent à des durées de phase de latence et de démarrage de l'activité acidifiante plus longues pour les laits microfiltré et pasteurisé par rapport au lait cru. Cela est à mettre en relation, pour les laits non ensemencés, avec le développement plus important des flores contaminantes des laits épurés (coliformes et psychrotrophes en particulier), et, pour les laits ensemencés, avec le développement moins important de la flore lactique dans le lait microfil- tré ; ce comportement peut s'expliquer par les modifications de substrat engendrées lors du traitement de microfiltration : on peut penser qu'une partie du potentiel biologique permettant l'élaboration des nutriments nécessaires au développement des bactéries lactiques est retenue par la membrane; par ailleurs, la nature de la flore résiduelle du lait microfiltré pourrait également avoir une incidence différente sur le développement de la flore acidifiante.

\section{Évolution des flores contaminantes pendant la transformation fromagère}

Les figures 2 et 3 représentent l'évolution du niveau de contamination en coliformes fécaux et en staphylocoques pendant la transformation du lait de chèvre en fonction du type de technologie utilisée et du niveau de contamination initial du lait. 


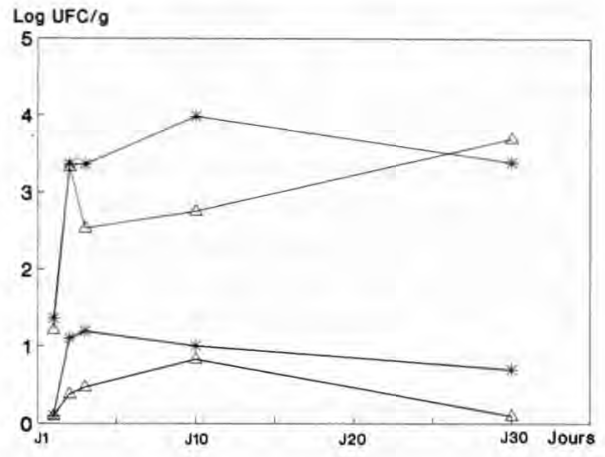

Fig 2. Évolution du niveau de contamination en coliformes fécaux pendant la fabrication et l'affinage de fromages de chèvre à pâte molle. * Technologie prise rapide ; $\Delta$ technologie prise lente ; - niveau de contamination initial inférieur à $1 \mathrm{Log}$ UFC/ml de lait ; - niveau de contamination initial supérieur à $1 \mathrm{Log}$ UFC/ml de lait.

Evolution in the level of faecal coliform contamination during cheese-making and ripening of soft goat's cheeses. * fast rennet coagulation ; $\Delta$ slow coagulation ; - initial level of contamination < 1 Log CFU/ml milk ; - initial level of contamination $>1 \mathrm{Log}$ CFU/mI milk.

Le niveau de population des flores contaminantes augmente pendant l'égouttage du coagulum, quels que soient le type de flore ou la technologie considérés.

Les taux multiplicatifs moyens respectivement observés pour les technologies prise rapide et prise lente sont de l'ordre de 1,5 et 1,0 unité pour la flore staphylocoque présumée pathogène et de 1,5 unité pour les coliformes fécaux. Ces observations rejoignent celles de Franck et Marth (1977) et Franck et al (1977), qui expliquent l'augmentation du niveau de contamination dans le fromage au démoulage par une concentration des germes (retenus dans le caillé) pendant l'égouttage, sans qu'il y ait multiplication importante des populations bactériennes. L'égouttage

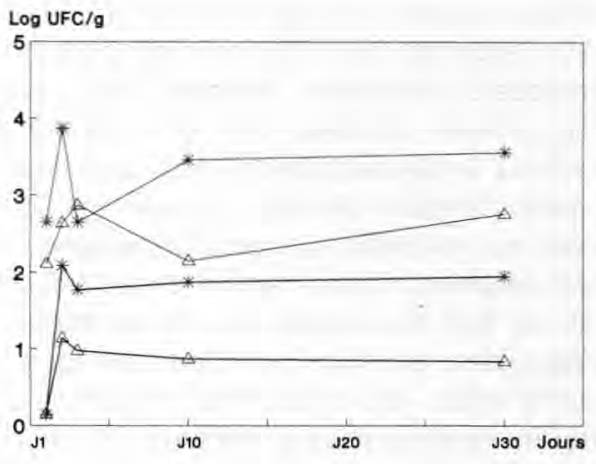

Fig 3. Évolution du niveau de contamination en staphycoloques pendant la fabrication et l'affinage de fromages de chèvre à pâte molle. * Technologie prise rapide ; $\Delta$ technologie prise lente ; - niveau de contamination initial inférieur à $1 \mathrm{Log}$ UFC/ml de lait ; - niveau de contamination initial supérieur à 2 Log UFC/ml de lait.

Evolution in the level of staphylococcal contamination during cheese-making and ripening of soft goat's cheeses. * fast rennet coagulation ; $\Delta$ slow coagulation; - inital level of contamination < 1 Log CFU/ml milk; - initial level of contamination $>2 \log$ CFU/mI milk.

étant plus rapide et brutal en technologie prise rapide, la concentration des germes serait plus importante, ce qui expliquerait en partie les niveaux de contamination plus élevés observés sur ces fromages par rapport à ceux fabriqués suivant une technologie prise lente.

Les niveaux de population des flores étudiées observés $30 \mathrm{j}$ après la fabrication sont comparables à ceux observés au stade démoulage, quels que soient la technologie étudiée et le niveau de contamination du lait mis en œuvre. Le développement des flores staphylocoque et coliforme fécale est lié aux conditions de conservation du fromage, et plus particulièrement à la température de stockage, mais aussi au $\mathrm{pH}$ du produit : Mourgues et al (1977) ont 
mis en évidence le rôle déterminant du $\mathrm{pH}$ sur l'évolution des coliformes dans les fromages à pâte molle ; Branger (1987) souligne l'effet inhibiteur d'un $\mathrm{pH}$ inférieur à 4,8 sur le développement des staphylocoques. Pendant l'affinage, ces germes peuvent se multiplier au fur et à mesure que le $\mathrm{pH}$ augmente sous l'action désacidifiante de la flore de surface. Le $\mathrm{pH}$ au démoulage étant plus bas pour les fromages à prise lente, $-0,5$ unité $\mathrm{pH}$ en moyenne, la durée nécessaire pour atteindre un $\mathrm{pH}$ de 4,8 est plus importante, ce qui expliquerait le niveau de contamination moyen plus faible observé sur ces fromages par rapport à ceux obtenus en technologie rapide.

Un faible niveau de contamination initial du lait en coliformes fécaux ( $<1$ UFC/ml) limite leur développement pendant la transformation fromagère : les populations observées après 10 et $30 \mathrm{j}$ de conservation sont inférieures à $10 \mathrm{UFC} / \mathrm{g}$ en moyenne, quelle que soit la technologie utilisée. Les staphylocoques présentent le même comportement en technologie prise lente ; en revanche, en technologie prise rapide, les populations observées pendant la conservation ne sont pas liées au niveau de contamination du lait mis en œuvre ; pour ces fabrications, les températures et les $\mathrm{pH}$ sont proches des conditions optimales de développement des staphylocoques (Branger, 1987) lors des phases de coagulation et d'égouttage. Ainsi, les niveaux de population observés dans les fromages au démoulage sont en moyenne supérieurs à $100 \mathrm{UFC} / \mathrm{g}$, pour des contaminations initiales inférieures à 10 UFC par $\mathrm{ml}$ de lait.

\section{CONCLUSION}

Les observations réalisées lors de cette étude montrent que la maîtrise d'un faible niveau de contamination en coliformes fécaux du lait de chèvre mis en fabrication permet de garantir un niveau de population acceptable dans les fromages à pâte molle, quelle que soit la technologie utilisée. Toutefois, il paraît indispensable de rechercher un développement rapide de la flore lactique acidifiante, de manière à limiter le.niveau de contamination en staphylocoques dans les fromages de chèvre à pâte molle, notamment en technologie prise rapide.

Les traitements de pasteurisation, $70^{\circ} \mathrm{C}$, $5 \mathrm{~min}$ et de microfiltration sur membrane $1,4 \mu \mathrm{m}$, présentent une efficacité comparable vis-à-vis des coliformes fécaux et des staphylocoques ; l'épuration quasi totale réalisée permet d'obtenir une qualité hygiénique satisfaisante du lait de chèvre destiné à la fromagerie.

Toutefois, ces traitements ne sont pas sélectifs et se traduisent également par une réduction importante de la flore sauvage du lait cru, qui nécessite un réensemencement en bactéries lactiques du lait épuré lors de la transformation fromagère. II apparaît par ailleurs que le traitement de microfiltration modifie le comportement du lait de chèvre pendant l'acidification ; permettant de réduire le niveau de dénaturation des protéines ; ce procédé d'épuration constitue une alternative intéressante à la pasteurisation du lait de chèvre ; toutefois son développement sera envisageable dans la mesure où la maîtrise des conditions de réensemencement du lait épuré permettront de réaliser une acidification comparable à celle observée sur le lait pasteurisé ou le lait cru.

\section{REMERCIEMENTS}

Ce travail a été réalisé dans le cadre d'un programme de recherche cofinancé par le ministère de l'Agriculture (DGAL), le ministère de la Recherche et de la Technologie, la région PoitouCharentes, I'ONILAIT, I'ANICAP, le BRILAC et les entreprises adhérentes à I'ITPLC. 


\section{RÉFÉRENCES}

Branger A (1987) Les staphylocoques présumés pathogènes dans les produits laitiers. Rev ENIL $114,30-39$ et $115,9-13$

Catteau M (1991) Les rapports de force entre les bactéries. Rev Lait Fr, suppl 512, 14

Frank JF, Marth EH (1977) Inhibition of enteropathogenic Escherichia coli by homofermentative lactic acid bacteria in skimmilk. J Food Prot 40, 754-759

Frank JF, Marth EH, Olson NF (1977) Survival of enteropathogenic and non pathogenic Escherichia coli during the manufacture of camembert cheese. J Food Prot 40, 835-842

Jaubert G, Costes P, Guyonnet P, Gay MF, Pierre A, Maubois JL (1991) Une épuration bactérienne à presque $100 \%$. Process 1066 , 62-67
Le Guillou S (1989) Pathologie mammaire et production laitière. Chèvre 174, 29-32

Mourgues R, Vassal L, Auclair J, Mocquot G, Vandeweghe J, Deschamps N, Vachot JC, Nicolas M (1977) Origine et développement des bactéries coliformes dans les fromages à pâte molle. Lait 57, 131-149

Portmann A, Pierre A (1973) Culture des ferments lactiques dans le lait de chèvre. Comparaison avec le lait de vache. Rev Lait Fr 312, 629-637

Richard J, Gratadoux JJ (1986) Facteurs affectant la croissance des bactéries coliformes dans les fromages à pâte molle. Sci Aliments 6, Hors série VI, 33-42

Zadow JC, Hardham JF, Kocax HR, Mayes JJ (1983) The stability of goat's milk to UHT processing. Aust J Dairy Technol 38, 20-23 